\title{
SOROPREVALÊNCIA DE Ehrlichia canis EM CÃES DE ABRIGOS E ASSOCIAÇÕES DE PROTEÇÃO, DO OESTE DO PARANÁ, BRASIL
}

Fransael Franklyn Araújo da Silva ${ }^{1}$ Anthony Henrique Cruz Da Silva ${ }^{2}$ Jaqueline Lunedo ${ }^{3}$ Leonardo Bianchi de Oliveira ${ }^{4}$ Simone Benghi Pinto ${ }^{5}$ Marivone Valentim Zabott ${ }^{6}$

SILVA, F. F. A. da; SILVA, A. H. C. da; LUNEDO, J.; OLIVEIRA, L. B. de; PINTO, S. B.; ZABOTT, M. V. Soroprevalência de Ehrlichia canis em cães de abrigos e associações de proteção, do Oeste do Paraná, Brasil. Arquivos de Ciências Veterinárias e Zoologia da UNIPAR, Umuarama, v. 23, n. 1 cont., e2312, 2020.

RESUMO: A erliquiose é uma doença riquetsial infecciosa causada por uma bactéria, pertencente ao gênero Ehrlichia, muito comum em cães e considerada uma importante hemoparasitose. Devido sua relevância na população canina e fácil transmissão por meio de carrapatos, mostra-se necessário conhecer melhor os aspectos epidemiológicos desta doença. Este estudo teve como objetivo avaliar a prevalência de Ehrlichia canis e os aspectos hematológicos em cães de abrigos temporários, de associações de proteção aos animais abandonados, nos municípios de Palotina e Guaíra, no Paraná. No total, 60 cães foram submetidos à coleta de amostras sanguíneas, para realização de hemograma e imunoflourescência indireta para detecção dos anticorpos anti-E. canis. A prevalência encontrada foi de $60 \%(36 / 60)$, não houve correlação positiva com relação ao sexo dos animais, presença de carrapatos ou trombocitopenia. Os dados revelam uma alta prevalência desta doença parasitária em cães de abrigos e a necessidade do estabelecimento de medidas de controle dos agentes transmissores.

PALAVRAS-CHAVE: Abrigos temporários. Erliquiose. Prevalência.

\section{SOROPREVALENCE OF Ehrlichia canis IN DOGS FROM SHELTERS AND PROTECTION ASSOCIATIONS IN WESTERN PARANÁ, BRAZIL}

\begin{abstract}
Ehrlichiosis is an infectious ricketsial disease caused by bacteria belonging to the Ehrlichia genus. It is very common in dogs and is considered as an important hemoparasitosis. Due to its relevance in the canine population and its easy transmission through ticks, it is necessary to further understand the epidemiological aspects of the disease. This study aimed at evaluating the prevalence of Ehrlichia canis and the hematological aspects in dogs from temporary shelters and associations for the protection of abandoned animals in the cities of Palotina and Guaíra, in the state of Paraná. Blood samples were collected from $60 \mathrm{dogs}$ for blood count and indirect immunofluorescence testing, and detection of anti-E. canis antibodies. A prevalence of 60\% (36/60) was found, with no positive correlation regarding the gender of the animals, presence of ticks, or thrombocytopenia. The data reveal a high prevalence of this parasitic disease in shelter dogs and the need to establish control measures related to the transmitting agents.
\end{abstract}

KEYWORDS: Temporary shelters. Ehrlichiosis. Prevalence.

\section{SOROPREVALENCIA DE Ehrlichia canis EN PERROS DE REFUGIOS Y ASOCIACIONES DE PROTECCIÓN, DEL OESTE DE PARANÁ, BRASIL}

RESUMEN: Ehrlichiosis es una enfermedad ricketsial infecciosa causada por una bacteria perteneciente al género Ehrlichia, muy frecuente en perros y considerada una importante hemoparasitaria. Debido a su relevancia en la población canina y fácil transmisión a través de garrapatas, es necesario conocer mejor los aspectos epidemiológicos de esta enfermedad. Este estudio tenía como objetivo evaluar la prevalencia de Ehrlichia canis, así como los aspectos hematológicos para la protección de animales abandonados en los municipios de Palotina y Guaíra, Paraná. Un total de 60 perros fueron sometidos a muestras de sangre para el recuento sanguíneo y la inmunoflourescencia indirecta detección de anticuerpos anti-E canis. La prevalencia encontrada fue del 60\% (36/60), sin correlación positiva con la el sexo de los animales, la presencia de garrapatas o trombocitopenia. Los datos revelan una alta prevalencia de esta enfermedad parasitaria en perros refugio y la necesidad de establecer medidas de control para los agentes transmisores.

PALABRAS CLAVE: Refugios temporales. Ehrlichiosis. Prevalencia.

DOI: 10.25110 /arqvet.v23i1cont.2020.7732

${ }^{1}$ Médico Veterinário, graduado pela Universidade Federal do Paraná - Setor Palotina. fransael20@gmail.com

${ }^{2}$ Acadêmico do curso de Medicina Veterinária, Universidade Federal do Paraná - Setor Palotina. anthony.henrique.pna@gmail.com

${ }^{3}$ Médica Veterinária, graduada pela Universidade Federal do Paraná - Setor Palotina. jaquelinelunedo2405@gmail.com

${ }^{4}$ Médico Veterinário, graduado pela Universidade Federal do Paraná - Setor Palotina. oliveiraleo1@gmail.com

${ }^{5}$ Bióloga. Doutora em Ciências Biológicas (UFPR). Docente do Departamento de Biociências da Universidade Federal do Paraná - Setor Palotina. sbenghi@ yahoo.com.br

${ }^{6}$ Médica Veterinária. Doutora em Zootecnia (UEM). Docente do Departamento de Biociências da Universidade Federal do Paraná- Setor Palotina. marvalentim@ufpr.br 


\section{Introdução}

A Erliquiose Monocítica Canina (EMC) é causada por uma bactéria intracelular estrita, gramnegativa denominada Ehrlichia canis, que parasita células hematopoiéticas especialmente do sistema fagocitário mononuclear, sendo transmitida principalmente pelo carrapato Rhipicephalus sanguineus e isso ocorre quando o vetor infectado realiza o repasto sanguíneo, o que mantém o microrganismo por transmissão transestadial uma vez que o mesmo é trixeno (BREMER et al., 2005).

A doença compreende três fases: fase aguda, quando ocorre a presença de bactérias na corrente sanguínea e tem como principais alterações hematológicas a anemia, trombocitopenia e leucopenia; fase subclínica, quando se observam alta titulação de anticorpos, com alterações hematológicas mais discretas, porém sem sintomatologia; e fase crônica, que ocorre quando o sistema imune do hospedeiro não consegue combater a infecção e apresenta sinais clínicos e laboratoriais mais evidentes (ANDEREG; PASSOS, 1999).

Os sinais incluem depressão, letargia, anorexia, pirexia e megalias, podendo os cães apresentar tendências a sangramento, principalmente petéquias e equimoses na pele $\mathrm{e}$ nas mucosas, sendo que os sinais oculares não são incomuns e incluem uveíte anterior e opacidade de córnea (WANER; HARRUS, 2000).

O diagnóstico clínico é o mais utilizado, ou seja, durante a rotina levam-se em consideração os sinais clínicos do paciente, presença ou não de carrapatos e as alterações hematológicas, sendo que segundo Aguiar (2006) entre os testes sorológicos a imunofluorescência indireta é o método laboratorial mais utilizado na detecção de anticorpos anti-E. canis, sendo aplicado em estudos de infecções experimentais.

O tratamento da erliquiose está baseado no uso de antibióticos de escolha como a doxiciclina, terapia suporte com fluidoterapia e transfusões sanguíneas, caso necessário, sendo que na ausência de vacinas disponíveis que protejam contra a doença, ressalta-se a importância do controle de carrapatos como medida de prevenção (PASSOS et al., 1999; WANER; HARRUS, 2000).

A erliquiose ocorre em muitos países de clima temperado, tropical e subtropical do mundo, coincidindo com a prevalência do seu vetor (ALMOSNY; MASSARD, 2002). É uma doença mundialmente distribuída em várias regiões geográficas, as quais incluem sudeste da Ásia, África, Europa, Índia, América Central e a América do Norte (WOLDEHIWET; RISTIC, 1993). No Brasil, a doença teve seu primeiro caso relatado em Belo Horizonte, MG em 1973 (COSTA et al., 1973), estando amplamente distribuída em todo o território nacional, principalmente no meio urbano (LABRUNA; PEREIRA, 2001).

Devido à ampla distribuição da erliquiose no país, alta casuística na clínica de pequenos animais, relatos de infecção em humanos e poucos estudos de prevalência da infecção em cães na região oeste do Paraná, ressalta-se a importância desta pesquisa, partindo-se da hipótese que a alta aglomeração de animais possa causar uma alta prevalência da doença na região.

Dessa forma, esse estudo teve por objetivo caracterizar a soroprevalência de Ehrlichia canis em cães de abrigos e associações de proteção animal do oeste do estado do Paraná, Brasil, através da imunofluorescência indireta.

\section{Material e Métodos}

O presente trabalho obteve aprovação da Comissão de Ética no Uso de Animais do Setor Palotina da Universidade Federal do Paraná (CEUA/Palotina), em 07/12/2016, protocolo $n^{\circ} 53 / 2016$. Foram utilizados na pesquisa 60 cães (26M; 34F) de abrigos temporários do oeste do Paraná. Os municípios estudados foram Palotina, situada a 341 metros de altitude, possuindo as coordenadas geográficas: $24^{\circ} 16^{\prime}$ $54^{\prime \prime}$ e $53^{\circ} 50^{\prime} 25^{\prime \prime}$ Oeste, onde foram coletadas amostras de 19 animais e Guaíra, a 245 metros de altitude, coordenadas: $24^{\circ}$ 4' $46^{\prime \prime}$ e $54^{\circ} 15^{\prime} 27^{\prime \prime}$, sendo coletadas amostras de 41 cães. Os municípios distam entre si aproximadamente $70 \mathrm{~km}$.

Os tutores dos animais receberam e assinaram o Termo de Consentimento Livre e Esclarecido (TCLE) e cada animal recebeu um número de identificação sendo preenchida uma ficha contendo informações sobre sexo, estado sanitário e nutricional destes. As coletas foram realizadas nos meses de junho e agosto de 2018, num total de 60 coletas sanguíneas, obtidas por venopunção jugular ou cefálica, divididas em duas amostras, uma com anticoagulante (EDTA) para realização de hemograma e outra com gel retentor de coágulo, para obtenção do soro, conservado a $-6^{\circ} \mathrm{C}$ até o momento da prova sorológica. A contagem das células sanguíneas foi realizada utilizando-se o analisador hematológico automático Mindray BC 2800 Vet ${ }^{\circledR}{ }^{7} \mathrm{e}$ a imunofluorescência indireta (IFI) utilizando-se o kit Imunodot $\AA^{8}$ de diagnóstico in vitro para erliquiose canina, sendo que as lâminas foram examinadas em microscópio de fluorescência Olympus FSX1007M. As análises foram realizadas nos Laboratórios de Parasitologia e Doenças Parasitárias, Laboratório Clínico e Laboratório de Patologia Animal do Setor Palotina da UFPR. Para avaliar as associações entre diferentes variáveis, utilizou-se o Teste do Quiquadrado $\left(\chi^{2}\right)$ corrigido por Yates, ou Exato de Fisher, quando algum valor esperado foi igual ou inferior a 5 . Considerou-se o valor $\mathrm{p} \leq 0,05$ estatisticamente significativo.

\section{Resultados}

A prevalência de cães positivos para $E$. canis dos abrigos de Palotina e Guaíra foi de 60\% (36/60). Os resultados de prevalência quando comparados entre os municípios não demostraram diferença significativa ( $\mathrm{p}>0,05$; Tabela 1).

Do total de animais sororreagentes 18 eram machos e 18 fêmeas $\left(\chi^{2}=1,626 ; p=0,201847\right)$. Dos 60 animais avaliados, apenas $13(21,7 \%)$ apresentaram infestação por carrapatos, destes, sete sororreagiram à IFI $\left(\chi^{2}=0,261 ; \mathrm{p}=\right.$ $0,608841)$. Com relação à contagem de plaquetas, 45 cães $(75 \%)$ apresentaram plaquetopenia $\left(<200.000 / \mathrm{mm}^{3}\right)$ e destes, 25 foram reagentes $\left(\chi^{2}=1,48 ; p=0,223543\right)$, portanto, não foi observada associação significativa $(\mathrm{p}>0,05)$ para esta e também para as demais variáveis hematológicas analisadas: número de hemácias, hematócrito e número de leucócitos (Tabela 1).

\footnotetext{
Mindray, São Paulo, SP

${ }^{8}$ Imunodot, Joboticabal, SP
} 
Tabela 1: Frequência (\%), valor de Quiquadrado $\left(\chi^{2}\right)$ e valor de $\mathrm{P}$ dos cães amostrados e reagentes à Imunofluorescência Indireta (título $\geq 64$ ) anti-Ehrlichia canis, em abrigos de Palotina e Guaíra oeste do Paraná, PR.

\begin{tabular}{lcccc}
\hline \multicolumn{1}{c}{ Variáveis } & Amostrados & Reagentes (\%) & $\boldsymbol{\chi 2}$ & P \\
\hline Machos & 26 & $18(69,2)$ & & \\
Fêmeas & 34 & $18(52,9)$ & 1,626 & 0,202 \\
\hline Palotina & 19 & $13(68,4)$ & & \\
Guaíra & 41 & $23(56,1)$ & 0,82 & 0,364 \\
\hline Ausência R. sanguineus & 47 & $29(61,7)$ & & \\
Presença R. sanguineus & 13 & $7(53,8)$ & 0,261 & 0,609 \\
\hline Plaquetas $\geq 200.000 / \mathrm{mm}^{3}$ & 15 & $11(73,3)$ & & \\
Plaquetas $<200.000 / \mathrm{mm}^{3}$ & 45 & $25(55,5)$ & 1,48 & 0,223 \\
\hline Hemácias $\geq 5,5 \mathrm{milhões} / \mathrm{mm}^{3}$ & 29 & $17(58,6)$ & & \\
Hemácias $<5,5$ milhões $/ \mathrm{mm}^{3}$ & 31 & $19(61,3)$ & 0,043 & 0,833 \\
\hline Hematócrito $\geq 37 \%$ & 33 & $18(54,5)$ & & \\
Hematócrito $<37 \%$ & 27 & $18(66,7)$ & 0,9054 & \\
\hline Leucócitos $\geq 6.000 / \mathrm{mm}^{3}$ & 58 & $35(60,3)$ & & 0,340 \\
Leucócitos $<6.000 / \mathrm{mm}^{3}$ & 2 & $1(50)$ & 0,0861 & 0,769 \\
\hline
\end{tabular}

Algumas alterações clínicas foram observadas em sete cães sororreagentes, incluindo (7) emagrecimento, (1) mucosas pálidas, (2) sinais dermatológicos. Apenas um animal apresentou hipertermia não sendo sororreagente.

\section{Discussão}

A positividade de anticorpos anti-E.canis encontrada nesta pesquisa (60\%) em cães de abrigos do oeste do Paraná, foi igual à observada por Almeida e Souza (2006), no município de Cuiabá, MT, mesmo sendo em cães atendidos em um hospital, utilizando o SNAP 3 DX e semelhante ao encontrado por Nakaghi et al. (2008), de 63,3\% (19/30), ao examinarem 30 animais suspeitos, utilizando IFI, atendidos no Hospital veterinário, UNESP, Jaboticabal, SP.

Os dados de prevalência desse estudo foram superiores aos encontrados por Silva et al. (2010) em Cuiabá (MT), de 42,5\% (108/254) em cães domiciliados; por Pereira (2015) em Palotina (PR), de 23,75\% (88/342) quando relatada em cães domiciliados sem sinais clínicos e por Trapp et al. (2006) em Londrina (PR), de 23\%(87/381), em cães atendidos em um hospital veterinário. Contudo, foi inferior ao obtido por pesquisadores utilizando IFI como: Azevedo et al. (2011) em Campos de Patos (PB), de 72,5\% (79/109), em cães com queixas variadas atendidos no Hospital veterinário da Universidade Federal de Campina Grande; Witter et al. (2013) em Cuiabá (MT), de 70,1\% (54/77), em animais com sintomatologia atendidos no HOVET-UFMT e por Meneses et al. (2008) em Salvador (BA), de 98,67\% (74/75), em cães com suspeita clínica.

Nesse estudo a diferença de frequências entre os municípios de Palotina e Guaíra não apresentou diferença significativa, acredita-se que por serem cidades próximas (70 $\mathrm{km}$ ), clima similar, datas de coletas próximas e animais em condição semelhante de origem e criação.

As variações na prevalência de E. canis estão relacionadas principalmente às condições climáticas, distribuição do vetor, práticas de manejo, ambiente de permanência dos animais, tipo de amostragem, tamanho das amostras, sensibilidade das técnicas e reações cruzadas principalmente no caso da sorologia (CARLOS et al., 2007; NAKAGHI et al.,2008; VIEIRA, 2011). Conforme citado por Witter et al. (2013), estudos epidemiológicos no Brasil constatam que a prevalência da EMC varia de 4,8\% no extremo Sul a 70,9\% no Centro-Oeste.

Como constatado por Silva et al. (2010) e Azevedo et al. (2011), animais com acesso somente intradomiciliar apresentaram uma frequência significativamente menor de infecção por E. canis do que aqueles com acesso ao peridomicílio, permitindo contato com animais de rua os quais não apresentam nenhum tratamento ou medida de combate ao vetor, facilitando a exposição a este e consequente a infecção. Devido ao clima subtropical úmido dos municípios estudados, os animais amostrados, serem oriundos de vida livre e encontrarem-se em associações de proteção e abrigos temporários, com alta densidade populacional, admite-se serem estas as principais causas para elevada prevalência da riquétsia nesse estudo.

Com relação à presença de $R$. sanguineus, Moreira et al. (2003) sugeriram que os cães expostos a carrapatos são mais propensos a apresentar níveis elevados de infecção por E. canis. Para Costa Junior et al. (2007), as alternâncias nas condições climáticas foram atribuídas como fatores importantes e que influenciam na dinâmica populacional dos carrapatos. Trapp et al. (2006), relataram a presença do carrapato como fator de risco associado à positividade para E. canis. Contudo, essa associação não foi observada por outros pesquisadores (UENO et al., 2009; SOUSA et al., 2010; WITTER et al., 2013; PEREIRA, 2015) assim como no presente trabalho, possivelmente devido as amostras terem sido coletadas no inverno, muito embora segundo Witter et al. (2013), como a positividade de anticorpos IgG reflete infecções passadas, não é necessário que o animal esteja infestado por carrapatos para ser sororreagente.

Não houve associação significativa com o sexo dos animais estudados e a positividade para $E$. canis corroborando com o observado por outros autores (FERREIRA et al., 2012; MANOEL, 2010; SILVA et al., 2010; WITTER et al., 
2013). Entretanto, em outras condições de experimentação, esses resultados diferem-se dos dados encontrados. Como foi para Pereira (2015), onde os machos apresentaram maior prevalência, explicado provavelmente pelo maior acesso à rua por parte destes e para Borin et al. (2009), que observaram um maior número de fêmeas positivas, justificado pelo maior número de atendimentos, não necessariamente relacionados à EMC.

Segundo Manoel (2010), anemia e a trombocitopenia podem ser observadas tanto na fase aguda como crônica da infecção por E. canis, sendo que a leucopenia é frequentemente observada em estágios terminais da infecção. Witter et al. (2013), ao avaliarem 77 cães sintomáticos suspeitos para EMC, bem como por apresentarem carrapatos durante a consulta, evidenciaram trombocitopenia e anemia associadas à positividade para erliquiose. Ferreira et al. (2012) ao analisarem cães atendidos em clínicas veterinárias em Jataí, GO, observaram alta frequência de trombocitopenia e anemia, porém sem associação com EMC, assim como no presente trabalho quando analisadas as variáveis hematológicas, apesar de ter ocorrido uma frequência elevada de animais com plaquetopenia (75\%), não houve associação significativa com a positividade para E. canis e nenhum animal positivo para a bactéria apresentou sinais clínicos sugestivos de EMC, muito embora havia relatos (verbais) de erliquiose clínica com diagnóstico e tratamento de alguns animais principalmente no período do verão.

\section{Conclusão}

Conforme resultados obtidos nesse estudo, concluise que houve elevada prevalência de anticorpos anti-E. canis em cães de associações de proteção e abrigos temporários e Palotina e Guaíra, PR, mesmo com uma baixa infestação por carrapatos, no momento da coleta e grande número de cães assintomáticos, demonstrando que há ampla distribuição da bactéria entre esses animais.

Faz-se necessária, portanto, uma maior atenção com a prevenção da erliquiose, nestes locais de alta aglomeração, no que diz respeito à domiciliação dos cães de vida livre, o controle do vetor nessa população, bem como medidas de controle populacional, tendo em vista diminuir a prevalência da doença e o risco ao ser humano, visto que existe um potencial zoonótico.

\section{Referências}

AGUIAR, D. M. Aspectos epidemiológicos da erliquiose canina no Brasil. São Paulo, 2006. Tese (Doutorado em Medicina Veterinária) - Universidade de São Paulo.

ALMEIDA, A. B. P. F.; SOUSA, V. R. F. Análise sorológica e biomolecular da infecção por Ehrlichia canis. Ciência Animal Brasileira, supl. 1, p. 265-267, 2006.

ALMOSNY, N. R. P.; MASSARD, C. L. Erliquiose em pequenos animais e como zoonose. In: ALMOSNY, N. R. P. Hemoparasitoses em Pequenos Animais Domésticos e como Zoonoses. 1 ed. Rio de Janeiro: L.F. Livros Ltda., 2002. p. 14-56.
ANDEREG, P. I.; PASSOS, L. M. F. Erliquiose canina: a revisão. Clínica Veterinária, v. 4, n. 19, p. 31-38, 1999.

DE AZEVEDO, S. S. et al. Soroprevalência e fatores de risco associados à soropositividade para Ehrlichia canis em cães do semiárido da Paraíba. Brazilian Journal of Veterinary Research and Animal Science, v. 48, n. 1, p. 14-18, 2011.

BORIN, S.; CRIVELENTI, L. Z.; FERREIRA, F. A. Aspectos epidemiológicos, clínicos e hematológicos de 251 cães portadores de mórula de Ehrlichia spp. naturalmente infectados. Arquivo Brasileiro de Medicina Veterinária e Zootecnia, v. 61, n. 3, p. 566-571, 2009.

BREMER, W. G. et al.Transstadial and intrastadial experimental transmission of Ehrlichia canis by male Rhipicephalus sanguineus.Veterinary Parasitology, v. 131, n. 1-2, p. 95-105, 2005.

CARLOS, R. S. A. et al. Frequência de anticorpos antiErhlichia canis, Borrelia burgdorferi e antígenos de Dirofilaria immitis em cães na microregião Ilhéus - Itabuna, Bahia, Brazil. Revista Brasileira de Parasitologia Veterinária, v. 16, n. 3, p. 117-120, 2007.

COSTA Jr., L. M. C. et al. Sero-prevalence and risk indicators for canine ehrlichiosis in three rural areas of Brazil. The Veterinary Journal, v. 174, n. 3, p. 673-676, 2007.

COSTA, J. O.; SILVA, M.; BATISTA Jr., J. A.; GUIMARÃES, M. P. Ehrlichia canis infection in dogs in Belo Horizonte - Brazil. Arquivos da Escola de Veterinária da Universidade de Minas Gerais, v. 25, p. 199-200, 1973.

DAGNONE, A. S. et al. Ehrlichiosis in anemic, thrombocytopenic, or tick-infested dogs from a hospital population in South Brazil. Veterinary Parasitology, v. 117 , n. 4, p. 285-290, 2003.

DOBROWOLSKI, E. C. et al. Soroprevalência e fatores de Riscos da Ehrlichia canis em cães da região de Guarapuava. In: CONGRESSO BRASILEIRO DE MEDICINA VETERINÁRIA E $1^{\circ}$ CONGRESSO SUL-BRASILEIRO DA ANCLIVEPA, 21., 2., 2016, Curitiba. Anais [...] Curitiba: 2015. p. 0034-0036.

FERREIRA, M. R. A. et al. Prevalência, fatores de risco e associações laboratoriais para erliquiose monocítica canina. Enciclopédia Biosfera, v. 8, n. 15, p. 1345-1356, 2012.

LABRUNA, M. B.; PEREIRA, M. C. Carrapato em cães no Brasil. Clínica Veterinária, São Paulo, Guara, v. 6, n. 30, p. 24-32, 2001.

MACIEIRA, D. B. et al. Prevalence of Ehrlichia canis infection in thrombocytopenic dogs from Rio de Janeiro, Brazil. Veterinary Clinical Pathology, v. 34, n. 1, p. 44-48, 2005. 
MANOEL, C. S. Alterações clínicas, hematológicas e sorológicas de cães infectados por Ehrlichia canis. São Paulo, 2010. Dissertação (Pós-Graduação em Clínica Veterinária) - Faculdade de Medicina Veterinária e Zootecnia. Universidade de São Paulo.

MENESES, I. D. S. et al. Perfil clínico-laboratorial da erliquiose monocítica canina em cães de Salvador e região metropolitana, Bahia. Revista Brasileira de Saúde e Produção Animal, v. 9, n. 4, p. 770-776, 2008.

MOREIRA, S. M. et al. Estudo retrospectivo (1998-2001) sobre erliquiose canina em Belo Horizonte, MG, Brasil. Arquivo Brasileiro de Medicina Veterinária e Zootecnia, v. 55, n. 2, p. 141-147, 2003.

NAKAGHI, A. C. H. et al. Canine ehrlichiosis: clinical, hematological, serological and molecular aspects. Ciência Rural, v. 38, n. 3, p. 766-770, 2008.

PEREIRA, P. Prevalência da ehrlichia spp. e da babesia spp. e fatores associados, em cães domiciliados em Palotina, Paraná, Brasil. Palotina, 2016. 65 f. Dissertação (Mestrado em Ciência Animal) - Setor Palotina, Universidade Federal do Paraná.

SANTOS, F. et al. Molecular evaluation of the incidence of Ehrlichia canis, Anaplasma platys and Babesia spp. in dogs from Ribeirão Preto, Brazil. The Veterinary Journal, v. 179, n. 1, p. 145-148, 2009.

SOUSA, V. R. F. Avaliação clínica, morfológica, hematológica, bioquímica e biomolecular de cães naturalmente infectados por Ehrlichia canis $e$ Anaplasma platys. Rio de Janeiro, 2006. 46 f. Tese (Doutorado) - Universidade Federal Rural do Rio de Janeiro.

SILVA, M. V. M. et al. Erliquiose canina: Revisão de Literatura. Arquivos de Ciências Veterinárias e Zoologia da UNIPAR, Umuarama, v. 14, n. 2, p. 139-143, 2011.

SILVA, J. N. et al. Soroprevalência de anticorpos antiEhrlichia canis em cães de Cuiabá, Mato Grosso. Revista Brasileira de Parasitologia Veterinária, v. 19, n. 2, p. 108-111, 2010.

TRAPP, S. et al. Seroepidemiology of canine babesiosis and ehrlichiosis in a hospital population. Veterinary Parasitology, Amsterdam, v.140, p.223-230, 2006.

UENO, T. E. H. et al. Ehrlichia canis em cães, atendidos em hospital veterinário de Botucatu, Estado de São Paulo, Brasil. Revista Brasileira de Parasitologia Veterinária, v. 18, n. 3, p. 57-61, 2009.

WANER, T.; HARRUS, S. Canine Monocytic Ehrlichiosis. In: CAMICHAEL, L. E. Recent Advances in canine infectious diseases, 2000. International Veterinary Information Service. Disponível em: https://pdfs. semanticscholar.org/b19e/15c84a975bac851dfce84c3d4c13 ed747 37f.pdf . Acesso em: 30 set. 2019.

WITTER, R. et al. Prevalência da erliquiose monocítica canina e anaplasmose trombocítica em cães suspeitos de hemoparasitose em Cuiabá, Mato Grosso. Semina: Ciências Agrárias, Londrina, v. 34, n. 6, p. 3811-3822, 2013.

WOLDEHIWET, Z.; RISTIC, M. Ricketsial and chamydial diseases of domestic animals. Pergamon Press, 1993. p. 427.

Recebido em: 28.11.2019 Aceito em: 02.10.2020 